Volumen: 30 Fecha de recibido: 8/1/2020

Año: 2020

Fecha de aceptado: 7/2/2020

Numero: 2 Número de páginas: 1-28
Correo: revista.sciencia@up.ac.pa

Fecha de publicación: Julio-Diciembre de 2020

URL: https://revistas.up.ac.pa/index.php/scientia

\title{
DETERMINACIÓN DE LA CAUSA RAÍZ DE LA CONTAMINACIÓN DIFUSA Y PUNTUAL DE LA CALIDAD DE AGUA EN BAHÍA MANZANILLO, CORREGIMIENTO DE CRISTÓBAL, DISTRITO Y PROVINCIA DE COLÓN, PANAMÁ
}

\section{DETERMINATION OF THE ROOT CAUSE OF DIFFUSE AND POINT POLLUTION OF WATER QUALITY IN BAHÍA MANZANILLO, CORREGIMIENTO DE CRISTÓBAL, DISTRICT AND PROVINCE OF COLÓN, PANAMA}

\author{
Javier Amir Hurtado Yow \\ Universidad de Panamá, Centro Regional Universitario de Colón, Panamá, \\ Panamá
}

\author{
javier.hurtado@up.ac.pa
}

\section{https://orcid.org/0000-0003-2662-8608}

\section{RESUMEN}

Uno de los problemas más grandes de las descargas de aguas residuales es que actualmente se desconoce su origen o causa raíz. Esta investigación trata de determinar la causa raíz de la contaminación difusa y puntual de la calidad de agua en bahía Manzanillo, corregimiento de Cristóbal, distrito y provincia de Colón de la República de Panamá. Se levantó un análisis geográfico de cuencas y microcuencas para determinar fuentes del problema hidrológico, basado en programas de mapeo del Modelo Digital de Terreno, o Digital Elevation Model (DEM) y la Misión Topográfica Shuttle Radar, o Shuttle Radar Topographic Mission (SRTM). En este estudio se comprueba el deterioro de los cuerpos de agua y la zona con mayor probabilidad de generar los vertimientos (o descargas), se identificó un polígono central como el prioritario para analizar y se infirió que dentro de esta unidad se encuentra la fuente contaminante de descargas de aguas residuales, donde se determinó que hay una contaminación puntual y no difusa. Se propone elaborar una herramienta para iniciar la restauración ecológica que queremos sea 
considerada como elemento clave a la hora de establecer las futuras redes de control de contaminación.

PALABRAS CLAVES: Agua, aguas residuales, causa raíz, contaminación puntual, Bahía Manzanillo.

\begin{abstract}
One of the biggest problems with wastewater discharges is that its origin or root cause is currently unknown. This investigation tries to determine the root cause of the diffuse and punctual pollution of the water quality in Manzanillo Bay, located in Cristóbal, Colón district and province, of the Republic of Panama. A geographic analysis of basins and micro-basins was carried out to determine sources of the hydrological problem, based on mapping programs of the Digital Elevation Model (DEM) and the Shuttle Radar Topographic Mission (SRTM). In this study, the fall of bodies of water and the area with the greatest probability of generating discharges is verified, a central polygon was identified as the priority to analyze and it was inferred that within this unit is the polluting source of wastewater discharges, where it was determined that there is a punctual and not diffuse contamination. It is proposed to develop a tool to initiate ecological restoration that we want to be considered as a key element when establishing future pollution control networks. This study applies a methodology to address a current problem that covers two main issues of sustainable development: water and environmental sanitation. The research is a great starting step for the proper management of environmental sanitation in the study area.
\end{abstract}

KEY WORDS: Water, wastewater, root cause, point pollution, Bahía Manzanillo.

\title{
INTRODUCCIÓN
}


La provincia de Colón cuenta con siete (7) cuencas hidrográficas registradas, a saber, cuenca del río Coclé del Norte (cuenca No. 105); cuenca de ríos entre Coclé del Norte y Miguel de la Borda (cuenca No. 107); cuenca del río Miguel de la Borda (cuenca No. 109); cuenca del río Indio (cuenca No. 111); cuenca de ríos entre el Indio y el Chagres (cuenca No. 113); cuenca del río Chagres o Cuenca del Canal de Panamá (cuenca No. 115); cuenca de ríos entre Chagres y Mandinga (cuenca No. 117), según Batista (2010).

La cuenca del río Chagres o Cuenca del Canal de Panamá (No. 115), ocupa el distrito de Colón, dentro del cual se localiza el corregimiento de Cristóbal, donde se encuentra la bahía de Manzanillo (UP, 1995). Es en esta bahía donde están ubicados los puertos de cruceros (o Home Ports) y las mayores terminales marítimas del país, que están en funcionamiento desde la década de los 90. Desde entonces, esta bahía ha sufrido la pérdida de cobertura boscosa de manglares en sus costas, así como contaminación que ha impactado a la fauna y flora acuática (Batista, 2001; von Storch, 2001; STRI, 2010; Grey \& Castillero, 2014).

Por otra parte, la contaminación no se ha detenido, ya que un volumen considerable de aguas residuales o servidas, domésticas e industriales, son vertidas a la bahía Manzanillo, las cuales aún persisten y se están incrementando mucho más, dado el crecimiento poblacional y las descargas no controladas de éstas, que no están siendo reparadas por las entidades competentes (Grey \& Castillero, 2014).

En el sector de Arco Iris Viejo, corregimiento de Cristóbal, distrito y provincia de Colón, se presentan diversos cuerpos de aguas sin previo estudio, particularmente, dentro de los predios del Centro Regional Universitario de Colón (C.R.U.C.) de la Universidad de Panamá, de los cuales se observan tres tipos de aguas, como (1) 
aguas servidas, que pasan por el C.R.U.C. a través de un sistema de cuneta, así como (2) aguas potables, las cuales llegan al C.R.U.C. proveniente de la potabilizadora de Monte Esperanza administrada por la Autoridad del Canal de Panamá (IDAAN, 2017), y (3) aguas estancadas, producto de los tipos anteriores o de lluvias (ETESA, 2017; NOAA, 2017), las cuales quedan acumuladas en ciertos espacios sin poder correr.

Uno de los problemas más grandes es el caso de las aguas residuales que atraviesan el área de la Universidad de Panamá (C.R.U.C.), ya que actualmente se desconoce su origen o causa raíz. Sin embargo, está afectando primeramente por olores molestosos a la comunidad universitaria (7 mil personas, aproximadamente) y que continúa su paso por la comunidad de Arco Iris viejo, para finalmente ser descargadas indiscriminadamente en la bahía Manzanillo, cercana al área de las bodegas de mercancía en France Field de la Zona Libre de Colón.

La quebrada con aguas cloacales atraviesa los terrenos de la Universidad de Panamá (C.R.U.C.) y desemboca en la bahía de Manzanillo sin tratamiento alguno, lo que ocasiona que las aguas de esta zanja presenten, según nuestra observación preliminar, afectación no sólo los organismos que la habitan y la belleza paisajística que componen el ecosistema (Odum et al., 1971), sino también exponiendo dentro del centro a la población estudiantil a ser impactada por vapores con olores molestosos y por organismos vectores de patógenos como los insectos.

En el proceso de cambio de la temporada seca a la temporada lluviosa se manifiesta una gran proliferación de insectos, evento que podría estar relacionado con el aumento de las descargas de aguas servidas que llegan al C.R.U.C. 
Esta situación que se acentúa día a día, sin solución inmediata, hacer pensar que podría traer como consecuencia directa el deterioro de la salud ambiental en los ecosistemas antes mencionados (Schultz, [s.f.]) y la pérdida paulatina de especies de fauna, como peces y macroinvertebrados (Jackson \& D'Croz, 2003; D'Croz \& Robertson, 2007; Guzmán \& Holst, 2007) y flora, como algas (Díaz, 2001; Batista, 2001; Robaina et al., 2008), importantes para la pesca artesanal (Jácome, 2001; Cortés \& León, 2002; Batista, 2010).

Según cifras que expone el sitio web del C.R.U.C., esta casa de estudios superiores cuenta con una población aproximada de siete mil $(7,000)$ personas, distribuidas en 14 hectáreas. El mayor riesgo al cual se exponen los estudiantes del C.R.U.C. está cerca de las aguas que están detrás del edificio conocido como "El Arca" o Facultades de Humanidades, de Derecho y Ciencias Políticas y de Administración de Empresas y Contabilidad. Esta zona abarca mayor concentración de estudiantes, que caminan por este área para dirigirse sus salones de clases.

El canal de aguas servidas está a cielo abierto sin ningún tratamiento. Entra directamente al C.R.U.C. y presenta fuerte olor a podredumbre en diferentes partes de su recorrido. Este canal atraviesa el C.R.U.C. hasta el final de sus límites y continúa afectando también a la comunidad de Arco Iris y, finalmente, desembocan en los ecosistemas de manglares de Bahía Manzanillo.

Esta investigación trata de determinar la causa raíz de la contaminación difusa y puntual de la calidad de agua en bahía Manzanillo, corregimiento de Cristóbal, distrito y provincia de Colón. 
Este estudio, aplica una metodología para abordar una problemática actual que abarca dos temas principales del desarrollo sostenible: agua y saneamiento ambiental. La investigación es un gran paso de inicio para el manejo apropiado en saneamiento ambiental de la zona de estudio (Letayf \& González, 1994).

El objetivo de esta investigación es determinar la causa raíz de la contaminación difusa y puntual de la calidad de agua en bahía Manzanillo. Como objetivo específico se desea reconocer en campo el estado de contaminación en los cuerpos de aguas presentes dentro de los predios del Centro Regional Universitario de Colón (C.R.U.C.), de la Universidad de Panamá, que desembocan en la bahía Manzanillo, en el corregimiento de Cristóbal.

\section{MATERIALES Y MÉTODOS}

\section{1. ÁREA DE ESTUDIO}

El área de estudio se localiza en la República de Panamá, provincia de Colón, distrito de Colón, corregimiento de Cristóbal, sector de Arco Iris (áreas revertidas), según ARI (1999), entre la avenida Randolph y la avenida Ahmad Miguel Waked, dentro de los predios y alrededores del Centro Regional Universitario de Colón, el cual forma parte de la Universidad de Panamá. Sus coordenadas geográficas de referencia son $9^{\circ} 21^{\prime} 35^{\prime \prime} \mathrm{N}$ y $79^{\circ} 54^{\prime} 02^{\prime \prime} \mathrm{O}$ 


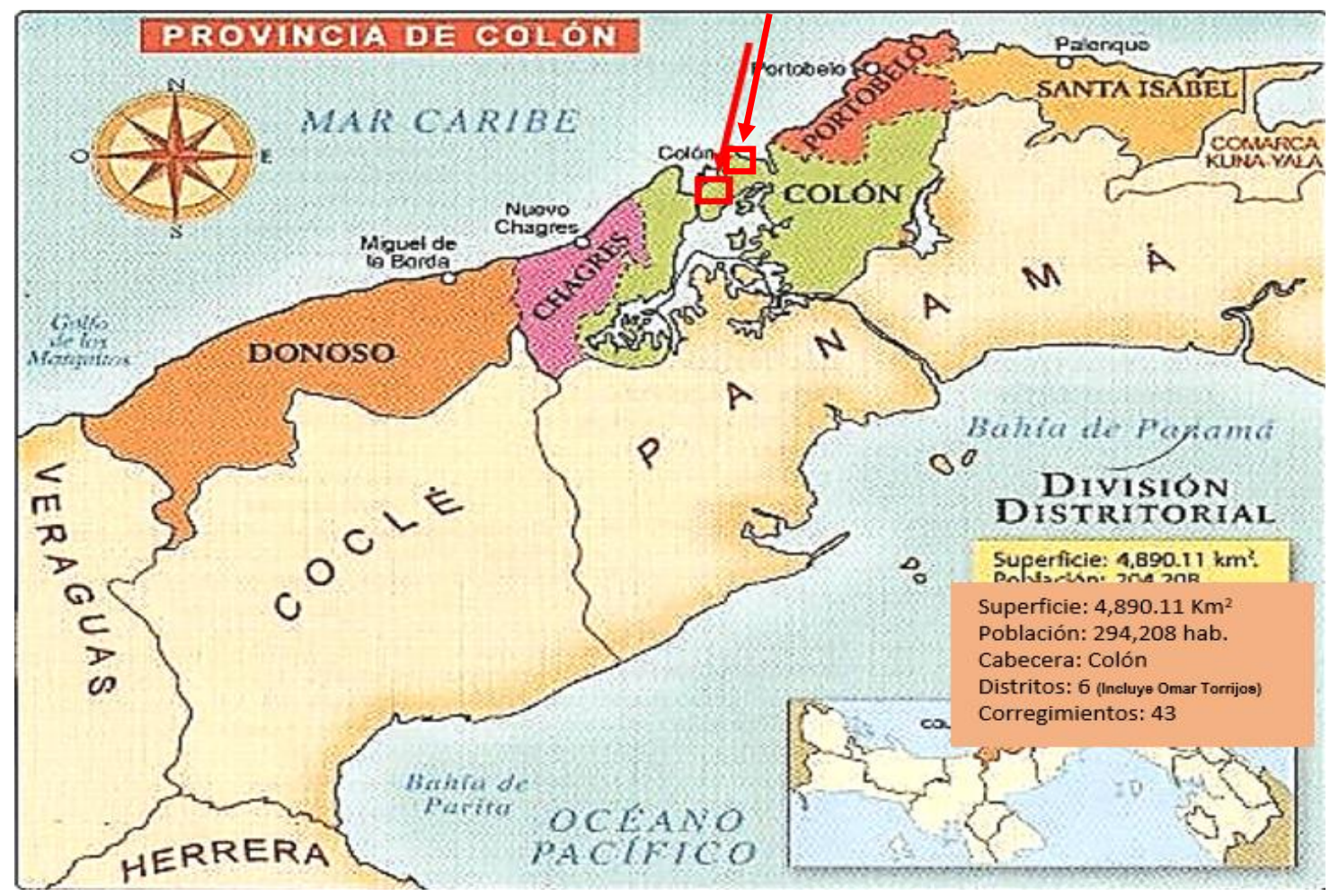

Imagen No.1: Mapa de la provincia de Colón

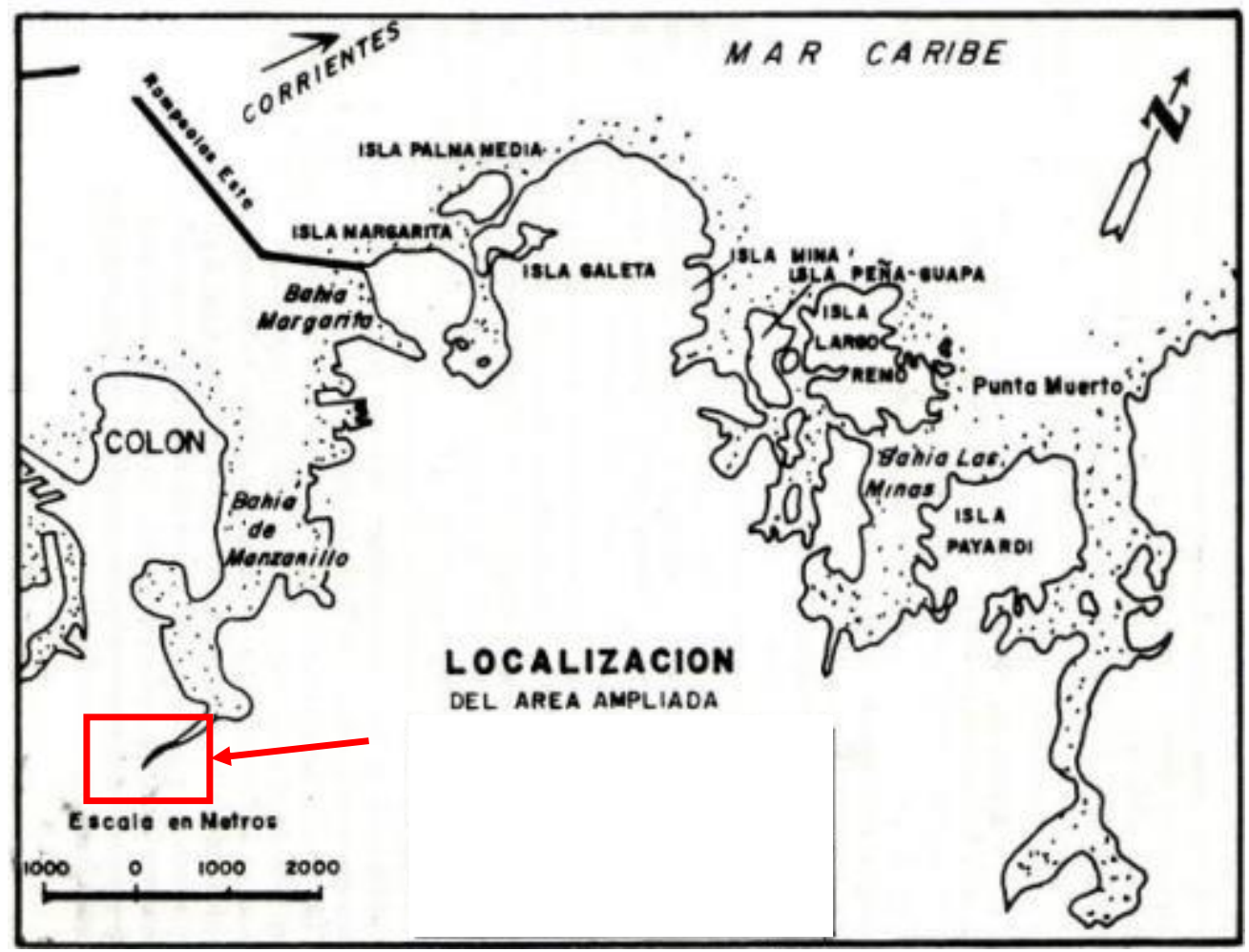

Imagen No.2: Localización del área de estudio (ampliada) 


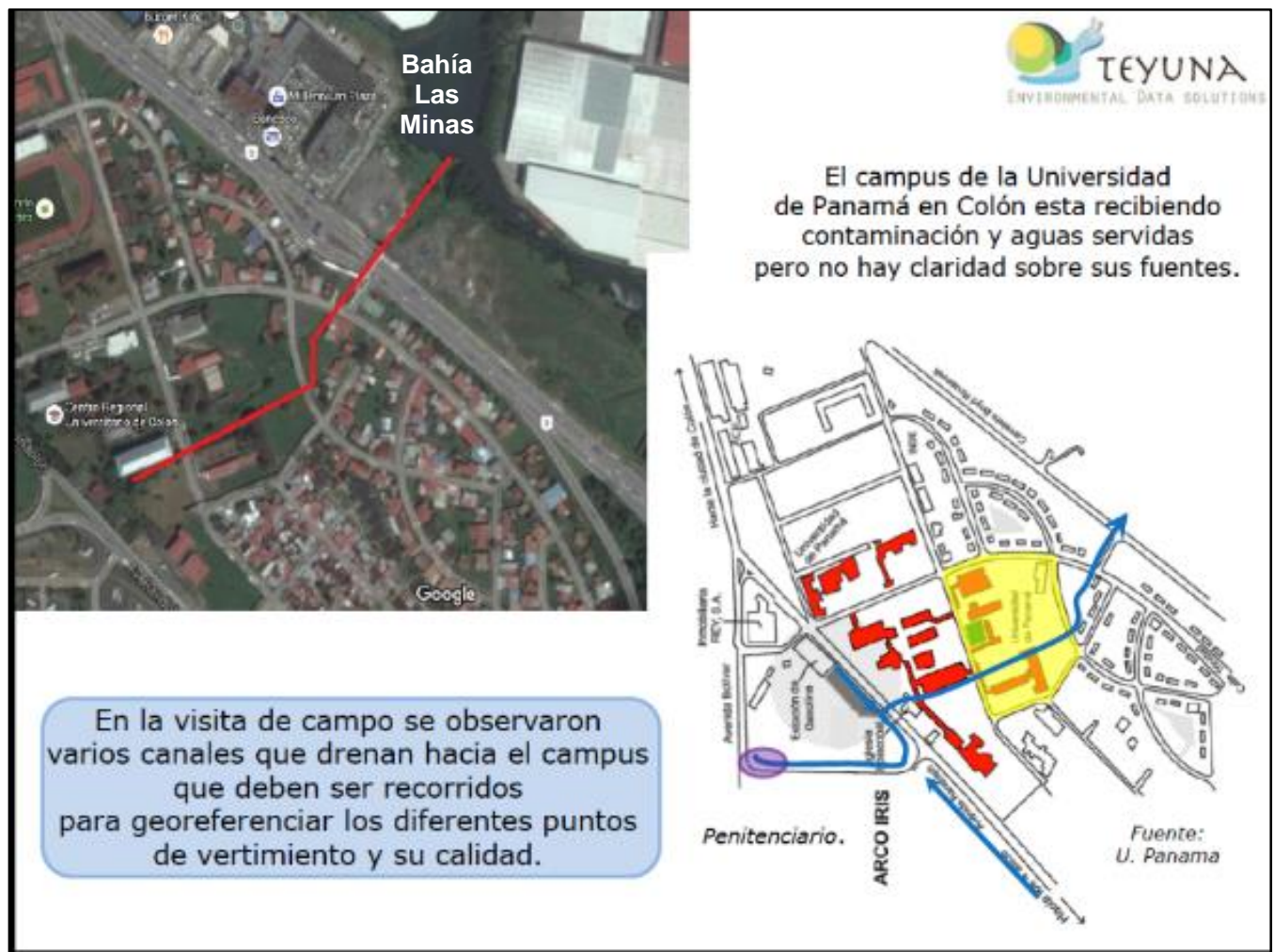

Imagen No. 3. Sitio específico de reconocimiento en campo

Fuente: Teyuna Environmental Data Solutions.

El sitio específico de reconocimiento en campo, correspondiente al cuerpo de agua estudiado, delimita al Norte con la comunidad de Arco Iris viejo, al Sur con la Avenida Randolph, al Este con la comunidad de Arco Iris nuevo y al $\mathbf{O}$ con la Avenida Randolph. Comprende una superficie total de 14 hectáreas, dentro de las cuales existe un cuerpo de aguas lóticas, conocido como Agua Sucia.

Es en un sitio compuesto de pastizales, rodeado de árboles de diversas especies siendo en su mayoría de árboles frutales y ornamentales; una fauna diversa de aves, insectos y reptiles (Contreras, et al., 2017). Las aguas de esta quebrada provienen de la Bahía Manzanillo través de un sistema de cunetas que a su vez se conecta con otro sistema pasando por el C.R.U-C y la comunidad de Arco Iris, 
terminando en los manglares presentes en el sector de la Bahía Limón.

\section{TIPO DE INVESTIGACIÓN:}

- La investigación es de tipo exploratoria que representa el primer acercamiento científico a este problema (Sampieri et al., 1998) y un estudio piloto de línea base sobre la determinación de la causa raíz de la contaminación difusa y puntual de la calidad de agua en bahía Manzanillo, corregimiento de Cristóbal, distrito y provincia de Colón.

- Se investigan por primera vez en el área y son estudios muy pocos investigados.

- Se emplean para identificar una problemática y familiarizarse con un problema desconocido, por lo que representa una situación de estudio innovador con un aporte nuevo en materia de investigación de ese tipo.

\section{POBLACIÓN Y MUESTRA}

Se reconocieron los cuerpos de agua en tres estaciones de muestreo, con una distancia aproximada entre cada una de 500 metros; ubicadas a lo largo de la cuneta de aguas pluviales impactada por aguas residuales, que atraviesa el Campus del Centro Regional Universitario de Colón (C.R.U.C.) de la Universidad de Panamá, con el fin de determinar el gradiente de distribución y concentración de los parámetros ambientales.

\section{FORMULACIÓN DE HIPÓTESIS}

$\mathrm{Ha}$

El estado de contaminación puntual y difusa sí afecta la calidad ambiental de los cuerpos de aguas presentes en el Campus Universitario de Colón de la 
Universidad de Panamá, los cuales desembocan en la Bahía de Manzanillo.

$H_{0}$

El estado de contaminación no afecta la calidad ambiental de los cuerpos de aguas presentes en el Campus Universitario de Colón de la Universidad de Panamá, los cuales desembocan en la Bahía de Manzanillo.

\section{VARIABLES}

- Variable independiente:

Niveles de concentración de contaminantes en los cuerpos de agua.

- Variable dependiente:

Afectación por contaminantes en los cuerpos de agua.

\section{INSTRUMENTOS DE RECOLECCIÓN DE DATOS}

- Utilización de programas de mapeo para determinar fuentes del problema hidrológico con un análisis geográfico de cuencas y microcuencas basado en un estudio fotogramétrico y sensores remotos a partir del Modelo Digital de Terreno, o Digital Elevation Model (DEM, por sus siglas en inglés) y técnicas de la Misión Topográfica Shuttle Radar, o Shuttle Radar Topographic Mission (SRTM, por sus siglas en inglés) de la Administración Nacional de Aeronáutica y el Espacio, o la NASA (acrónimo en inglés de National Aeronautics and Space Administration).

- El SRTM con el fin de obtener un modelo digital de elevación de la zona de estudio de modo que genere una completa base de mapas topográficos digitales de alta resolución.

- El DEM, con el fin de analizar el mapa hidrológico con imágenes para identificar las correctas elevaciones en las zonas de estudio que provocan 
el recorrido de las aguas y las llevan hasta bahía Manzanillo, para elaborar una guía de este recorrido.

\section{PROCEDIMIENTO METODOLÓGICO PARA DESARROLLAR EL OBJETIVO ESPECÍFICO:}

Se siguieron los siguientes procedimientos para reconocer en campo el estado de contaminación en los cuerpos de aguas presentes dentro de los predios y alrededores del centro regional universitario de Colón, de la Universidad de Panamá, que desembocan en la bahía Manzanillo, en el corregimiento de Cristóbal.

- Durante el periodo de los años 2016 y 2018, se realizaron visitas de campo con el apoyo del uso de sistemas de información geográfica para el manejo territorial de recursos hídricos, al área del C.R.U.C., así como sus alrededores, que previamente había sido identificada por los estudiantes de un curso de limnología, durante un semestre de clases, como área de gran peligro para las personas que allí conviven.

- Se establecieron los puntos con un GPS cuando se realizó el recorrido por el área para hacer un estudio rápido del estatus del área y polígonos alrededores.

- En el recorrido en campo, se realizó el reconocimiento del estado ecológico de los cuerpos de agua o ecosistemas acuáticos (Odum et al., 1971) impactados por aguas residuales (U.Y., 2018), donde se hizo un registro fotográfico, a lo largo de toda la línea de aguas servidas que en su recorrido atraviesan los predios del Centro Regional Universitario de Colón, de la Universidad de Panamá, que, a su vez, impactan la bahía Manzanillo, en Colón.

- Se levantó un análisis geográfico de cuencas y microcuencas basado en fotogrametría y sensores remotos a partir del Digital Elevation Model (DEM) y 
Shuttle Radar Topographic Mission (SRTM), con apoyo de la Organización Teyuna Environmental Data Solutions, que permitió ubicar el área de estudio en un contexto hidrológico para poder ubicar las fuentes del problema y descartar otras hipótesis.

- Descripción breve del sitio seleccionado para el reconocimiento del estado ecológico de los ecosistemas acuáticos:

Para el reconocimiento de aguas, se seleccionó un sitio dentro del C.R.U.C. el cual se localiza detrás de la facultad de Turismo éste presentaba tres tipos de aguas: Servida y Estancada. Es un lugar abierto con un suelo arcilloso con presencia de diversas especies de árboles, arbustos y herbáceas; al igual que una fauna variable compuesta de aves, insectos y reptiles.

\section{RESULTADOS Y DISCUSIÓN}

\section{RESULTADOS PARA OBJETIVO ESPECÍFICO:}

a) El análisis geográfico de cuencas y microcuencas basado en fotogrametría y sensores remotos a partir del Digital Elevation Model (DEM) y Shuttle Radar Topographic Mission (SRTM), permitió ubicar el área de estudio en un contexto hidrológico para poder hallar las fuentes del problema y descartar otras hipótesis.

b) Se evidencia que la causa raíz de la contaminación es muy puntual (o localizado), de la misma manera se descarta unidades hidrológicas que no drenan hacia el centro regional universitario de Colón ni tampoco afectan la calidad del agua de la bahía de Manzanillo.

c) La zona con mayor probabilidad de generar los vertimientos (o descargas) de aguas residuales se ubicó en el polígono central como el prioritario para analizar y se infirió que dentro de esta unidad se encuentra la fuente contaminante de 
descargas de aguas residuales, donde se determinó que hay una contaminación puntual (y no difusa), cuyo punto específico de origen se origina por colapso del tanque séptico del Centro Penitenciario Nueva Esperanza, administrado por la Dirección General del Sistema Penitenciario del Ministerio de Gobierno, República de Panamá.

d) Las aguas servidas provienen de un sistema de cunetas del Centro Penitenciario Nueva Esperanza y entidades como ENSA, estación de combustible y otras, que se han establecido cerca del área de la universidad (C.R.U.C.).

e) Los polígonos laterales, se descartaron al tener pequeñas laderas que aportan escorrentía superficial con poca probabilidad de estar drenando contaminantes, hacia los canales por su topografía y configuración hidrológica.

f) Al realizar el recorrido en campo para el reconocimiento del estado ecológico de los cuerpos de agua (ecosistemas hídricos) impactados por aguas residuales, donde se hizo un registro fotográfico, a lo largo de toda la línea de aguas servidas que en su recorrido atraviesan los predios del Centro Regional Universitario de Colón, de la Universidad de Panamá, que, a su vez, impactan la bahía Manzanillo, en Colón.

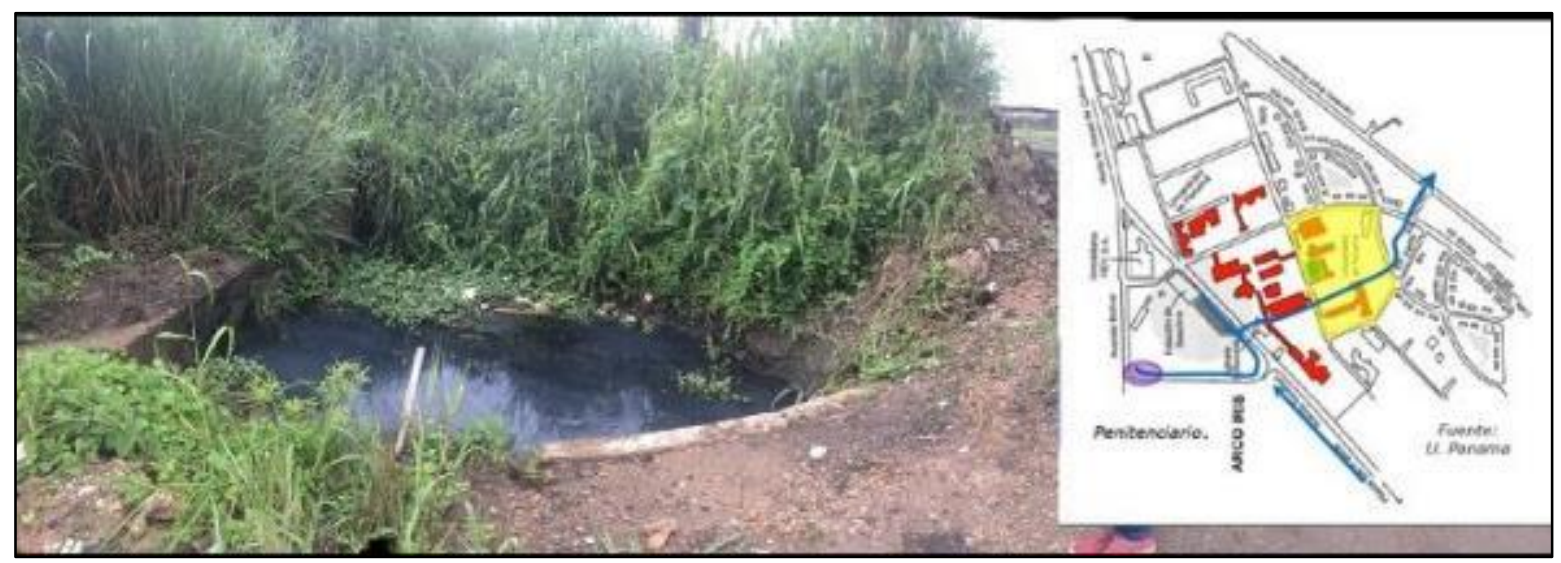

Imagen No. 4. Hallazgo de contaminación puntual, cuyo punto específico se origina por colapso del tanque séptico del Centro Penitenciario Nueva Esperanza, 
administrado por la Dirección General del Sistema Penitenciario del Ministerio de Gobierno.

Las observaciones en campo demuestran que existen impactos a los ecosistemas hídricos que se manifiestan en alarmas indicando que ya están a su mayor capacidad para soportar la contaminación proveniente de las grandes volúmenes de aguas residuales domésticas e industriales, con canales que presentan impactos acumulativos por cunetas improvisadas sin amortiguar los cauces naturales de los ecosistemas hídricos que tratan de llegar al mar por cualquier vía sin armonizar los procesos naturales cercanos a ambientes urbanos (como en la Universidad de Panamá), como zanjas construidas sin planeamiento ocasionando estancamientos de los cuerpos de agua que intentan llegar al mar.

Finalmente, impacto exponencial se ve demostrado en la bahía Manzanillo, santuario natural que garantiza las especies marinas de importancia comercial; sin embargo, la desembocadura del cuerpo de agua en estudio trastorna los procesos ecológicos naturales de esta zona.

\section{OTROS HALLAZGOS:}

1. Existe un deterioro de los suelos cercanos al área de estudio, con impactos a los ecosistemas hídricos que se manifiestan en inundaciones, inclusive en temporada seca cuando no existen frecuentes lluvias.

2. Se percibe que el corregimiento de Cristóbal, específicamente, en el sector de Arco Iris, está predestinado a seguir sufriendo continuas inundaciones y contaminaciones a causa de un mal planeamiento de los ecosistemas hídricos provenientes de las áreas residenciales, industriales y comerciales que garantizan la economía del país y de la región. 
3. Actualmente, se continúa sin corregir los cauces hídricos que se requieren para armonizar los ecosistemas.

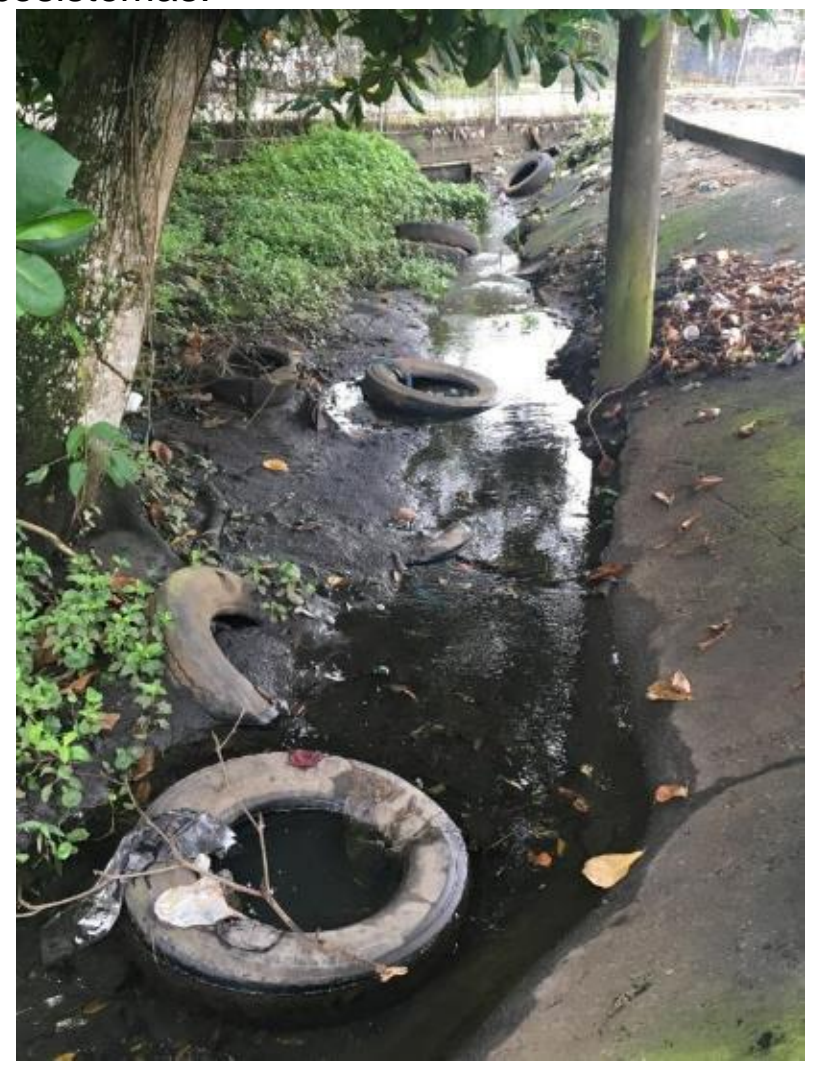

Imagen No. 5. Hallazgo. Diversos tipos de contaminación provenientes de la disposición incorrecta de los desechos sólidos traídos por las inundaciones y diversas actividades humanas. 


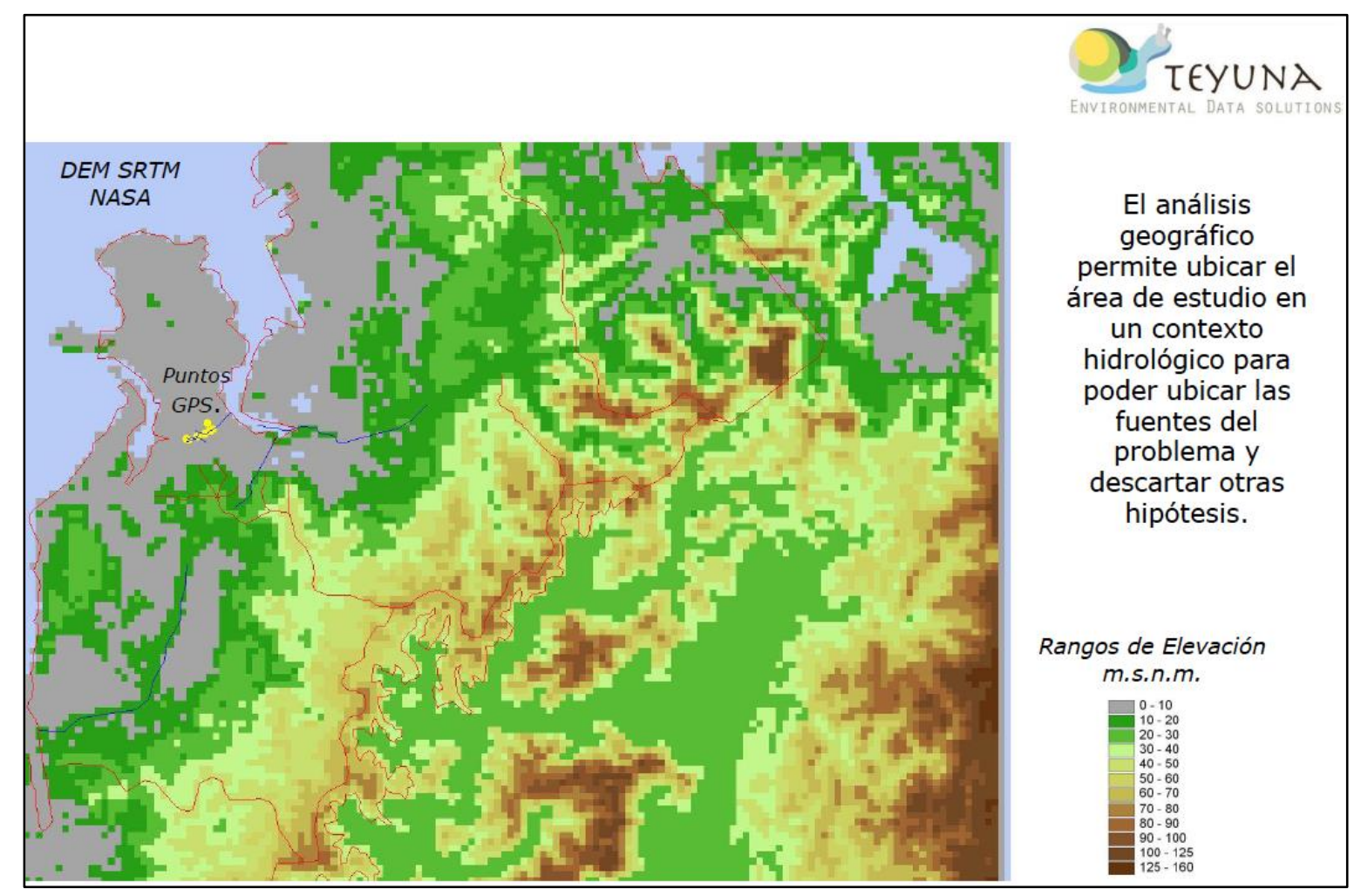

Mapa No. 1. Puntos GPS (Global Positioning System) demarcados en color amarillo y líneas azules (cuerpos de agua), sobre la zona de color gris 0 , la cual corresponde al rango de elevación que va de los 0 a 10 metros sobre el nivel del mar (m.s.n.m.), proporcionado por este análisis geográfico de cuencas y microcuencas basado en el Digital Elevation Model (DEM) y Shuttle Radar Topographic Mission (SRTM). Fuente: Teyuna Environmental Data Solutions. 


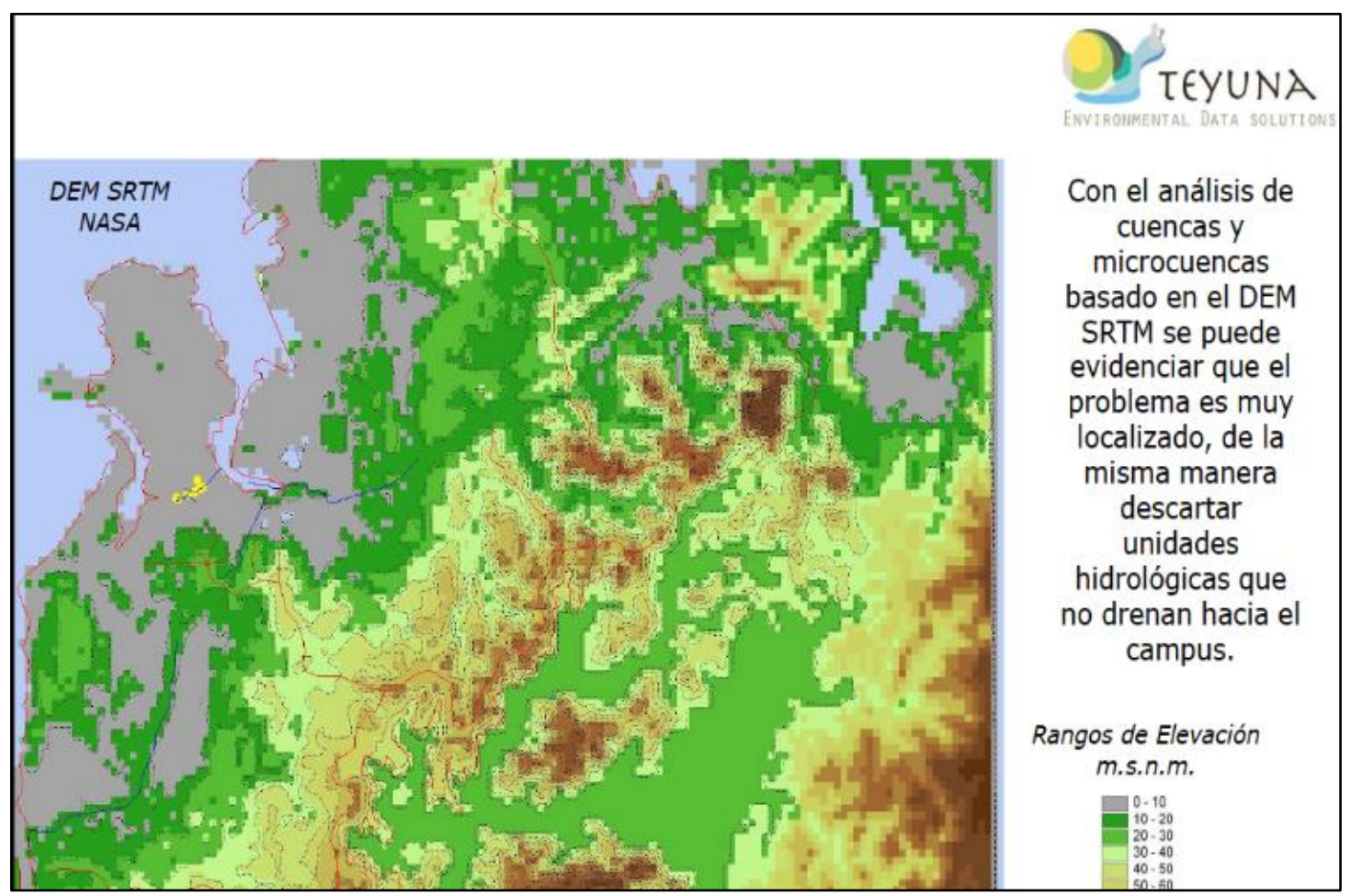

Mapa No. 2. Rangos de elevación en metros sobre el nivel del mar (m.s.n.m.) según análisis geográfico de cuencas y microcuencas basado en el Digital Elevation Model (DEM) y Shuttle Radar Topographic Mission (SRTM). Fuente: Teyuna Environmental Data Solutions. 


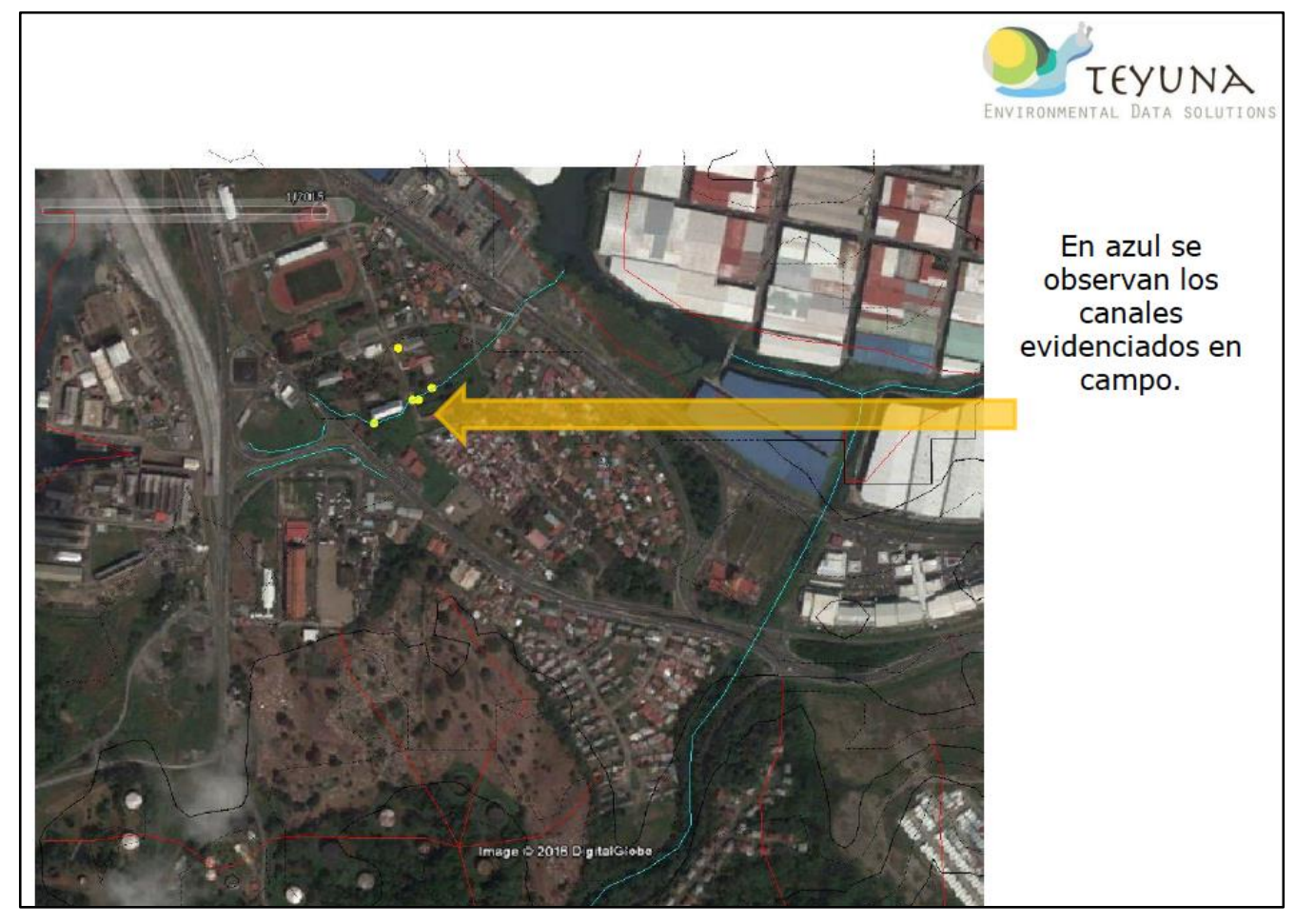

Imagen No. 6. Análisis geográfico de cuencas y microcuencas basado en el Digital Elevation Model (DEM) y Shuttle Radar Topographic Mission (SRTM). Fuente: Teyuna Environmental Data Solutions. 


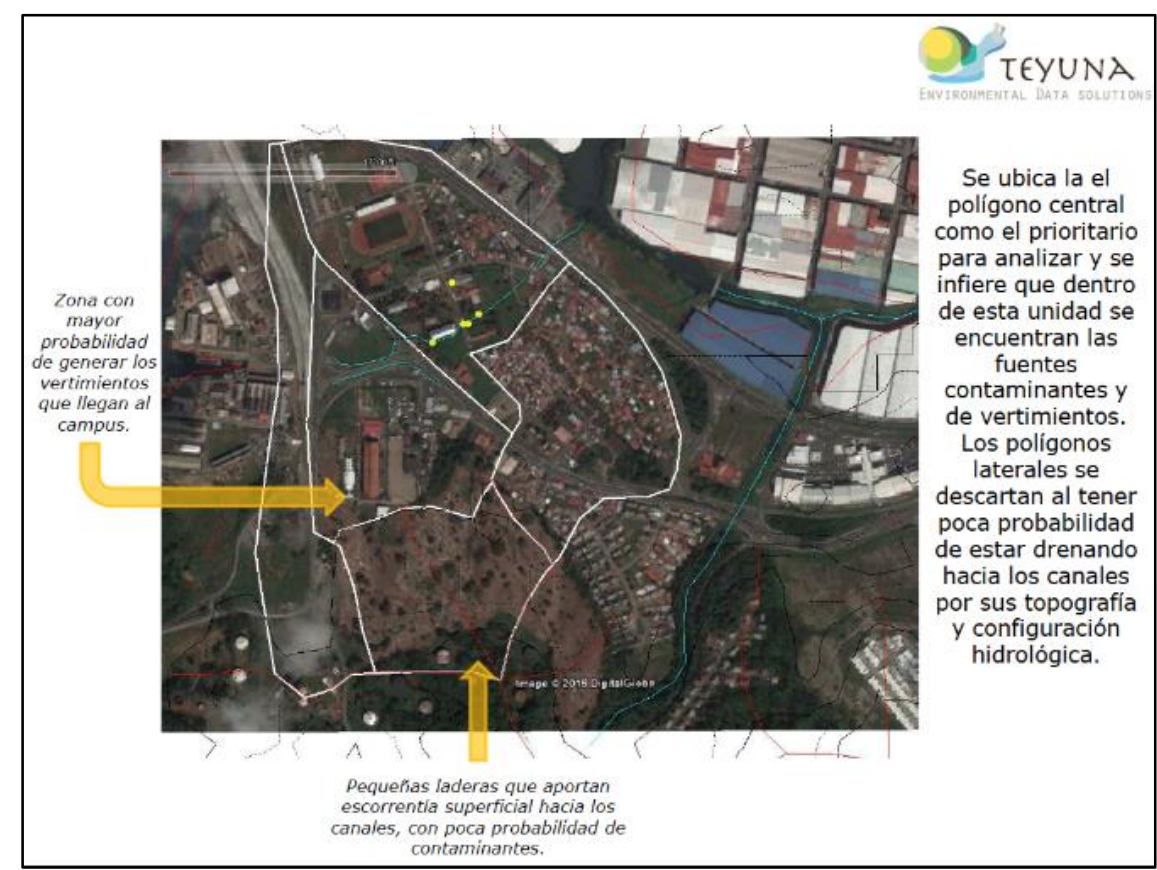

Imagen No. 7. Análisis geográfico de cuencas y microcuencas basado en el Digital Elevation Model (DEM) y Shuttle Radar Topographic Mission (SRTM). Fuente: Teyuna Environmental Data Solutions. 


\section{ANÁLISIS Y DISCUSIÓN DE RESULTADOS:}

a. Según el análisis geográfico de cuencas y microcuencas basado en el Digital Elevation Model (DEM) y Shuttle Radar Topographic Mission (SRTM), que se muestra en la Imagen No. 5, la zona con mayor probabilidad de generar los vertimientos (o descargas) se ubicó el polígono central como el prioritario para analizar y se infirió que dentro de esta unidad se encuentra la fuente contaminante de descargas de aguas residuales.

b. Las observaciones del estudio confirman que las fuentes son muy locales, identificando las elevaciones de los terrenos con mayor aporte por donde fluye el agua.

c. Se determinó que hay una contaminación puntual (y no difusa), cuyo punto específico de origen se ocasiona por colapso del tanque séptico del Centro Penitenciario Nueva Esperanza, administrado por la Dirección General del Sistema Penitenciario del Ministerio de Gobierno.

d. Como se puede observar en el análisis DEM y SRTM, se logró evidenciar que la causa raíz de la contaminación es muy puntual (o localizado), de la misma manera se descartaron unidades hidrológicas que no drenan hacia el centro regional universitario de Colón ni tampoco afectan la calidad del agua de la bahía de Manzanillo.

e. Los puntos GPS (Global Positioning System) demarcados en color amarillo y líneas azules (cuerpos de agua), sobre la zona de color gris, la cual corresponde al rango de elevación que va de los 0 a 10 metros sobre el nivel del mar (m.s.n.m.), proporcionaron información puntual que reflejó que cualquier tipo de cuerpo de agua, así sea pluvial, potable o residual, que sea descargada en esta zona, va a buscar drenar al mar, por cualquiera que sea la vía, según este 
análisis geográfico de cuencas y microcuencas basado en el Digital Elevation Model (DEM) y Shuttle Radar Topographic Mission (SRTM).

\section{CONCLUSIÓN}

El análisis de los resultados evidenciados en este estudio establece las condiciones de referencias para ser entregados a los docentes y administrativos de la Universidad de Panamá, así como a los administrativos del Centro Penitenciario de Nueva Esperanza de la Dirección General del Sistema Penitenciario del Ministerio de Gobierno de Panamá. Estos resultados son cruciales dentro de todo el proceso para la amortiguación de la corriente de agua y sus contribuyentes, ya que son la base para que la Universidad de Panamá y el Ministerio de Gobierno puedan elaborar una petición formal al Gobierno Nacional. Propuesta que dé la pauta para elaborar un plan de gestión que permita que en las áreas del cuerpo de agua en estudio, como las zonas aledañas, se establezcan proyectos de saneamiento ambiental y monitoreo costero, conservación y educación ambiental que garanticen el cuidado de esta zona costera de gran interés económico (de Vega, 1995; Medcoast Institute, 2004; Jackson, 2007; de Vega, 2014).

Como se estableció en la hipótesis, el estado de contaminación puntual y difusa sí afecta la calidad ambiental de los cuerpos de aguas presentes en el Campus Universitario de Colón de la Universidad de Panamá, los cuales desembocan en la Bahía de Manzanillo.

Los cuerpos de agua que han estado contaminados durante muchos años han generado un grave problema por la desaparición de los sustratos naturales en éstos y la acumulación de lodos como resultado de elementos muy tóxicos que afectan la biota, incluyendo a la población humana (STRI, 1987; 2010). 
En este estudio se comprueba el deterioro de los cuerpos de agua y propone que la Universidad de Panamá y demás instituciones involucradas en la gestión y saneamiento ambiental de éstos, jueguen un papel responsable para elaborar una herramienta para iniciar la restauración que queremos sea considerada como elemento clave a la hora de establecer las futuras redes de control de descargas de aguas residuales hacia bahía Manzanillo.

Estos hallazgos justifican que se elabore un plan estratégico que permita establecer un programa de monitoreo y manejo ambiental, en cumplimento a las normativas legales establecidas en esta materia cuidando esta zona costera de gran potencial económico y vital para todos los que habitan y trabajan especialmente cerca de estos lugares (Letayf \& González, 1994; Medcoast Institute, 2004).

Producto de los resultados obtenidos, se recomienda que se entregue una copia de esta investigación al Ministerio de Gobierno de Panamá, con copia a su Dirección General del Sistema Penitenciario, así como al Centro Penitenciario Nueva Esperanza, en Colón, para que se tomen las medidas necesarias para subsanar la causa raíz de la contaminación puntual (o localizada), de la calidad de agua en Bahía Manzanillo, corregimiento de Cristóbal, distrito de Colón y provincia de Colón.

Es imprescindible hacer el reconocimiento de otros puntos de vertimiento de las urbanizaciones vecinas en el corregimiento de Cristóbal, en otros canales dentro del polígono central que muestran las figura 6 y 7 .

Además de estos puntuales hallazgos fue impresionante ver desde las imágenes de satélite un relleno de cuerpos de agua y la tala de la última cobertura boscosa de mangle en la zona (Cubit, 1985; Batista, 2001), para ser reemplazada por concreto y bodegas, esto puede ser el foco de futuras inundaciones en toda el área. 
Este estudio es un gran paso de inicio para el manejo apropiado en saneamiento ambiental de la zona de estudio. La tarea más importante ahora es la de seguir detallando en el campo y preguntando a los actores sociales locales.

\section{REFERENCIAS BIBLIOGRÁFICAS}

ARI. Autoridad de la Región Interoceánica. Resolución Administrativa N²83-99 del 25 de agosto de 1999. Panamá, República de Panamá. 4 págs.

Batista de Vega, Gloria. 2010. Un vistazo a un ecosistema único en el mundo. Curso de BIO 331. Escuela de Biología. Universidad de Panamá. Panamá.

Batista de Vega, Gloria. 2001. Defendamos Isla Margarita: Patrimonio Natural $r$ Histórico de Colón, Panamá. Nemesix. Panamá, República de Panamá. 48 p.

Batista de Vega, Gloria. 2010. Buscando harmonía en los ecosistemas acuáticos. Curso de Limnología - BIO 330. Escuela de Biología. Universidad de Panamá. Panamá.

Bennett, D. P., \& Humphries, D. A. (1974). Introduction to field biology. Edward Arnold.

Contreras, M., Herrera, J., Bryand, G., \& Loredon, Y. (2017). Riqueza y abundancia de aves en el Centro Regional Universitario de Colón, Panamá. Revista Colón Ciencias, Tecnología y Negocios.

Cortés, José y Alberto León. 2002. Arrecifes coralinos del Caribe de Costa Rica. Editorial INBio. Instituto Nacional de Biodiversidad. Costa Rica. 136 p.

Cubit, John; et al. 1985. El valor de los manglares y arrecifes en la costa de Colón. Editado por Stanley Heckadon Moreno y Jaime Espinoza G. Impretex, S.A. Panamá, República de Panamá. Págs. 183-199.

D'Croz, Luis y D. Ross Robertson. 2007. Condiciones oceanográficas costeras que afectan a los arrecifes de coral en ambos lados del Istmo de Panamá. In: Jr. Leigh, Egbert Giles; Edward Allen Herre; Jeremy B.C. Jackson; Fernando 
Santos-Granero (Ed.), Ecología y evolución en los trópicos. p. 309-317. Editora Nova Art. Panamá.

Jackson, Jeremy y Luis D'Croz. 2003. El océano se divide. En Paseo pantera: una historia de la naturaleza y cultura de Centroamérica, de Anthony G. Coates. Smithsonian Institution Press. Washington, D.C. Págs. 41-79.

Díaz, Marco. 2001. El impacto del turismo en los bosques y arrecifes panameños. En Panamá: Puente Biológico de Stanley Heckadon Moreno. Instituto Smithsonian de Investigaciones Tropicales. Panamá, República de Panamá. P. 214-219.

ETESA, EMPRESA DE TRANSMISIÓN ELÉCTRICA S.A. 2017. Datos diarios de precipitación. Panamá, República de Panamá.

- http://www.hidromet.com.pa/datos_diarios.php

Grey, A., Domínguez, V., \& Castillero, M. (2014). Determinación de Indicadores Fisicoquímicos y Microbiológicos de calidad del agua superficial en la Bahía de Manzanillo. I+ D Tecnológico, 10(1), 16-27.

Guzmán, Héctor M. e Irene Holst. 2007. Efectos de la contaminación crónica por petróleo crudo en la reproducción del coral Siderastrea siderea en el Caribe. En: Ecología y Evolución de los Trópicos. Instituto Smithsonian de Investigaciones Tropicales. Panamá, República de Panamá. Págs. 645-653.

INSTITUTO DE ACUEDUCTOS Y ALCANTARILLADOS NACIONALES (IDAAN). 2017. Consultas: Departamento de Distribución. Dirección Regional de Colón.

Jackson, Jeremy. 2007. Los Arrecifes desde Colón. En Ecología y Evolución de los Trópicos. Instituto Smithsonian de Investigaciones Tropicales. Panamá, República de Panamá. P. 633-644.

Jácome, Gabriel. 2001. La pesca y el deterioro de los arrecifes en el Caribe y Panamá. En Panamá: Puente Biológico de Stanley Heckadon Moreno. Instituto Smithsonian de Investigaciones Tropicales. Panamá, República de Panamá. P. 200-206.

Letayf, J., \& González, C. (1994). Seguridad. Higiene y Control Ambiental, Editorial Mcgraw-Hill, México. 
Medcoast Institute. 2004. Coastal Systems II: Fundamentals of coastal and marine ecosystemology I and II. Program. Dalyan, Aegean Coast, Turkey

○ http://www.medcoast.org.tr/resources/institute_04_r.htm

NOAA, National Oceanic and Atmospheric Administration. 2017. National Weather Service > Climate Prediction Center $(\mathrm{CPC})>$ Monitoring and Data > Monitoring Atlantic Hurricane Potential.

○ http://www.cpc.ncep.noaa.gov/products/hurricane/

Odum, E. P., Odum, H. T., \& Andrews, J. (1971). Fundamentals of ecology(Vol. 3). Philadelphia: Saunders.

Robaina R., Rafael; García J., Pilar; y Batista de Vega, Gloria. 2008. Cultivo Ecosostenible de Algas Marinas (SEAWEED ECOFRIENDLY AQUACULTURE. SEA Panamá). Memoria PCI C5054/06 y Manuales de laboratorio. Universidad de Las Palmas de Gran Canaria y Universidad de Panamá. 137 págs.

Robertson, Ross. 2001. Arrecifes y peces de la costa pacífica panameña: una región biológica única. En Panamá: Puente Biológico de Stanley Heckadon Moreno. Instituto Smithsonian de Investigaciones Tropicales. Panamá. Págs. 39-44.

Sampieri, Roberto Hernández, Carlos Fernández Collado, Pilar Baptista Lucio, and Ma de la Luz Casas Pérez. Metodología de la investigación. Vol. 1. México: Mcgraw-hill, 1998.

Schultz, Arnold. [s.f.]. What is Ecosystemologysts? Berkeley.edu/classes. Universidad de Berkeley, California, U.S.A. 8 p.

- http://nature.berkeley.edu/classes/ecosystemology/what\%20is\%20ecosy stemology.pdf

STRI, Smithsonian Tropical Research Institute. 2010. LOS RESULTADOS DEL PROYECTO DEL DERRAME DE PETRÓLEO DE STRI PUEDEN LLEGAR A SER MUY ÚTILES. NOTICIAS SMITHSONIAN TROPICAL RESEARCH INSTITUTE. PANAMÁ, REPÚBLICA DE PANAMA. May 24, 2010.

○ http://www.stri.org/espanol/acerca_stri/noticias/noticias/articulo.php?i $\mathrm{d}=817$ 
STRI, Smithsonian Tropical Research Institute. 2010. Científicos del Smithsonian reportan altas temperaturas, blanqueamiento de corales y mortandad de invertebrados en Bocas del Toro. Información Pública y Medios de Comunicación. Fri, 1 Oct 2010.

STRI, Smithsonian Tropical Research Institute. 1987. Effects of an oil spill at Bahía Las Minas, Republic of Panama : proposal for research and addendum. Smithsonian Tropical Research Institute. Ancon, Panama. 229 págs.

STRI, Smithsonian Tropical Research Institute. 2010. The Galeta Oil Spill Project. Panama, República de Panamá.

o http://biogeodb.stri.si.edu/oilspill

von Storch, Hans. 2002. Good governance - synergy of science and coastal development.ppt (Power Point Presentation). Institute for Coastal Research Germany. Germany.

- 202 - 5.6.2002 Chamber of Commerce, Colon, Panama. Good governance - synergy of science and coastal development

- http://coast.gkss.de/staff/storch/talks.htm

UA, University of Arizona. 2010. PLN 500 Ecosystemology for Urban Planning. Environmental/Healthy Cities Planning Concentration. Master of Science in Planning. Arizona. United States.

○ http://www.planning.arizona.edu/program2.html

ULg, Université de Liège. 2010. Ecosystemology and environmental management course. Zoogeography Research unit, Institut de Botanique. Université de Liège. Belgique.

○ http://progcours.ulg.ac.be/archives/20072008/1C-

2C/cocoon/en/cours/ZOOL0215-1.html

UNICAUCA, Universidad del Cauca. Modelación deductiva de procesos naturales y antrópicos en ecosistemas tropicales (Módulo 1: Introducción a la Ecosistemología). Departamento de biología. Facultad de Ciencias Naturales, Exactas y de la Educación. Universidad del Cauca. Colombia. - http://dtm.unicauca.edu.co/maestelematica/EcosistemasTropicales.pdf 
UP, Universidad de Panamá. Departamento de Medio Ambiente, Tecnología Aplicada, \& Estudios Geográficos. (1995). Reflexiones ambientales en el 1a interoceánica (No. 9). Universidad de Panamá, Instituto del Canal de Panamá y Estudios Internacionales.

UY, Universidad de Yacambú. (2018). Apuntes sobre contaminación ambiental: la contaminación del agua. Venezuela.

- https://sites.google.com/a/micorreo.uny.edu.ve/contaminacionambiental/contaminacion-del-agua-1

DE VEGA, G. B. (1995). CONSIDERACIONES AMBIENTALES SOBRE LAS BASES MILITARES DE ESTADOS UNIDOS EN PANAMÁ. Las bases militares $y$ el desarrollo nacional, 2, 93.

DE VEGA, G. B. (2014). DEFENDAMOS ISLA MARGARITA "PATRIMONIO NATURAL E HISTÓRICO DE COLÓN, PANAMÁ". Revista Colón Ciencias, 1(2), 4581. 


\section{AGRADECIMIENTO.}

Resalto las seguridades de mi más distinguido respeto y las más sinceras muestras de agradecimiento a mi Profesora, la Doctora Gloria Batista de Vega, Biotecnóloga, quien con su alto nivel de compromiso ambiental sembró en mí la curiosidad intelectual para desarrollar este proyecto; asimismo, el apoyo del Dr. Roberto Carlo Jaramillo, Ecólogo, especialista en el uso de Sistemas de Información Geográfica, S.I.G., para el manejo territorial de recursos hídricos, junto al apoyo de la Organización Teyuna Environmental Data Solutions, que permitió ubicar el área de estudio en un contexto hidrológico para poder ubicar las fuentes del problema y descartar otras hipótesis, para la culminación exitosa de éste y cada uno de mis proyectos. 